\title{
Safer Interactive Medical Device Design: Insights from the CHI+MED Project
}

\author{
Paul Curzon \\ Queen Mary University of \\ London \\ London, UK \\ p.curzon@qmul.ac.uk
}

[Invited Paper]

\author{
Ann Blandford \\ UCL \\ London, UK \\ a.blandford@ucl.ac.uk
}

\author{
Harold Thimbleby \\ Swansea University \\ Swansea, UK \\ h.thimbleby@swansea.ac.uk
}

\author{
Anna Cox \\ UCL \\ London, UK \\ anna.cox@ucl.ac.uk
}

\begin{abstract}
Interactive medical devices such as infusion pumps, monitors and diagnostic devices help save lives. However, they may also fail in use and patient harm ensue. It is not just that the software and hardware should meet their specification. The design should help ensure users do not make mistakes. Safety factors are more important as medical devices become mobile and are used by patients as part of their everyday life rather than by trained professionals in well-defined hospital environments. Regulators are increasingly taking home-use seriously as a result of device recalls due to devices that have caused patient harm. We give insights from the research on the CHI+MED project (www.chi-med.ac.uk). It has focussed on understanding how the design of interactive medical devices can support safety. CHI+MED also developed practical tools and guidance that we review.
\end{abstract}

\section{Categories and Subject Descriptors}

H.1.2 [Information Systems]: User/Machine Systemshuman factors; K.4.1 [Computers and Society]: Public Policy Issues-human safety; J.3 [Life and Medical Sciences]: Medical Information Systems

\section{General Terms}

Design, Human Factors

\section{Keywords}

Safety, Medical Devices, Interaction Design

\section{INTRODUCTION}

From 2005-2010, in the US alone, 56,000 adverse events were reported with infusion pumps, with 710 deaths and 87 model recalls of unsafe designs. The recall of one pump is estimated to have cost its manufacturers $\$ 400-600$ million. Adverse events also cost health services in the effort spent on investigations, compensation, and pressure on the staff involved. Even if adverse events do not result, poor interaction design can lead to clinicians wasting time better spent on patient care. These issues apply to all interactive medical devices. Hospitals are busy, stressful places with clinicians constantly multitasking and often interrupted. People make mistakes in such environments. Good interaction design can help detect and prevent mistakes and hence prevent harm occurring when mistakes inevitably are made. Ill-thought-out design can, by contrast, increase the chances that mistakes are made. Safety factors become more important as medical devices become mobile and are used by patients as part of their everyday life rather than by trained professionals in well-defined hospital environments. Wireless and mobile technology have the potential to support innovative design solutions but only if interaction design is taken into account.

The CHI+MED project brought together researchers from UCL, Swansea, QMUL and City Universities to investigate safer design of interactive medical devices. The project focussed on four main areas: understanding normal use of devices in hospitals and the home; safe data entry; tools and guidance; and understanding incidents. In this paper, we overview a selection of the contributions and highlight insights from these themes relevant to mobile devices.

\section{UNDERSTANDING NORMAL USE}

Accident investigations and research into errors in healthcare focus on what went wrong. We have investigated 'normal' practice: what actually happens day-in-day-out, getting the job done and making patients better - when things go right. This includes not only understanding the way things are done, but also the potential for mistakes to happen, and how error can be, and is, avoided on a daily basis. It is important that normal practice is understood so that appropriate design decisions can be made, and as a basis for evaluating whether innovative medical device design appropriately supports the tasks being done. It also matters in understanding where errors emerge from, as well as issues 
that are broader than error and safety such as how the experience of the person using the device can influence its use. We have studied the everyday use of devices in settings such as on hospital wards, in people's home and on the move.

\subsection{Resilience strategies}

Better understanding and sharing of the ways people adapt what they do to avoid making mistakes can make healthcare safer. We developed the concept of 'resilience strategies': the informal and inventive actions people take to avoid making mistakes and improve performance [2]. We have created a categorisation scheme for resilience strategies and developed frameworks for analysing and understanding them including how and why they work. We are using this knowledge to better understand how patients and healthcare practitioners self-manage. This will lead to improvements in the design and use of medical devices.

\subsection{Patients' home experiences}

We interviewed patients who use haemodialysis at home about their experiences. Our work shows that the design of future machines needs to better take into account aspects of the process people find difficult, and fitting dialysis into their lives. Design recommendations include that real-time remote monitoring and remote intervention by technicians in case of emergency, as well as easy data and information exchange between patients and clinicians, are needed [14].

\subsection{Situated methods for mobile design}

Medical devices are increasingly part of patients' daily lives. New methods are needed to ensure they are easy to use in non-medical contexts. CHI+MED has investigated the use of Type 1 Diabetes technologies in people's everyday lives using qualitative situated methods. These methods go beyond the scope of the current human factors standards such as ISO 62366 . We are now applying them to the design and development of new diabetes technologies [12].

\subsection{Fieldwork for healthcare}

Fieldwork for healthcare is a very challenging research area, for which there was little support: a gap we have now filled. We have made it easier for new researchers and practitioners to do healthcare fieldwork studies by collecting case studies and experiences from international experts [4]. We have also identified guidance and strategies for overcoming the problems of doing fieldwork for healthcare [5].

\section{SAFER DATA ENTRY}

Entering data correctly, whether numbers or text, is a routine part of healthcare. Examples include entering details into patient records, setting drug doses, recording prescriptions, and so on. There is plenty of scope for mistakes that harm patients. The wrong information could be entered, but also the wrong sequence of steps might be followed. We have explored the underlying reasons why mistakes happen and how they can be avoided. We have looked at both the limitations of our cognition, and how changes to the design of data entry systems can help prevent such errors. Entering numbers is an important part of this. The purpose of any number entry interface is simple - to select a specific numeric value. The interfaces used can however be deceptively complicated as we have shown in a range of devices. Worse, the way they are implemented ignores user error and can result in unpredictable behaviour that could harm patients.

\subsection{Evaluating number entry interfaces}

We have evaluated number entry interfaces showing that the style of interface influences the type of error committed and its severity. There is a trade-off between speed and safety. Digit-based keypads are fast but likely to lead to severe errors, whereas so-called 5-key interfaces are slower but less likely to lead to dangerous mistakes. When keying errors are made, like trying to increase the number beyond the largest possible value, they should be blocked with a warning that should be acknowledged [13].

Some numbers that medical workers type into devices used on hospital wards are more common than others. Familiar numbers are faster to type than non-familiar ones. We have shown that usability studies need to take account of whether numbers used are familiar to the participants to reflect the real tasks performed. Entering numbers can be made easier if the interface is tailored based on the most commonly used numbers for the intended task.

\subsection{Interruptions}

Interruptions tax short term human memory, making error more likely. Our experiments have shown that if users take a few moments to recall what they were doing before they are interrupted, their memory is more accurate. Well-designed visual reminders support short term memory and make it easier to pick up where people left off and finish the task correctly [1].

\subsection{Focussing on the numbers}

Other experiments show that asking people questions linked to a number they are about to enter makes it more likely that they will do so correctly. It can also help them correct mistakes. In particular, people made far fewer errors after being asked questions about the format of the number, its quantity, and the context of the number entry task [6].

\subsection{Using control theory to model interaction}

We developed a new approach for evaluating user interfaces of medical devices based on techniques from aerospace engineering and robotics, combining manual control theory with hybrid automata. We modeled both discrete and continuous human operator behaviour and found a novel way to model a person using a medical device with up and down buttons. We can model both short button presses and holding down buttons. A typical insight is the identification of user interface design flaws that would lead to a large overshoot when adjusting numbers. It also helps an analyst to identify and reflect on different types of user behaviour [11].

\subsection{Programming safer keyed data input}

We have developed a tool that automatically creates data entry user interfaces that are safer to use than those created in ad hoc ways. It helps ensure programmers cover all eventualities and the interface responds sensibly to errors.

Programmers have at best ad hoc solutions over what to do when people enter data incorrectly, like typing a second decimal point or putting a leading 0 in a number. Often 
programmers just ignore these seemingly simple problems. Unfortunately, failing to manage user error well causes even worse problems for users. Our tool helps prevent this. One approach the tool uses is traffic lights, so the user interface and the user know when a mistake has been made. A red traffic light — perhaps augmented with a sound or vibration (and it could be any colour, or flash for colour-blind users) - alerts the user that a problem must be solved. With the tool, traffic lights are implemented flexibly, efficiently and dependably. Crucially, the programmer can then build software that does not need to handle the user errors, since the tool has done that already [16].

\section{TOOLS AND GUIDANCE}

An important aim of CHI+MED was not just to undertake scientific research but also to develop practical support for professionals based on that research. The support comes in a variety of forms such as guidance documents, practical techniques and resources that support them including computer based tools. We developed tools and guidance for a range of purposes throughout the project including: to support the design and procurement of new medical devices; to support the case arguing that a device is safe and satisfies regulatory requirements, as well as to check such claims; and to document and to analyse medical incidents.

\subsection{Prototyping and verification}

Our prototyping tool, PVSio-web [9], helps check the safety of designs of devices and in doing so it supports designers, regulators, and hospital training and procurement staff. Working with the US regulator, the Food and Drug Administration (FDA), it has helped identify problems in commercial medical devices. Hospitals have used our tool as part of training programmes highlighting safety-related design issues. It has a flexible, extensible architecture. It also includes support for modelling, prototyping and simulating interoperable devices, allowing their safety to be checked

\subsection{Hazard analysis for user interfaces}

Working with the FDA we also identified a substantial set of hazards related to the use of the data entry systems of infusion pumps. We determined how poor design could lead to these hazards and identified marketed devices where they occur. We have also developed systematic analysis techniques for identifying use-related hazards [8].

\subsection{Refinement in developing requirements}

Criteria for the acceptable safety of medical devices, safety requirements, are typically described precisely but in natural language. An important issue is how a regulator can be assured that the given requirements are satisfied. We have developed a refinement based approach that relates to the pre-market review process as promoted by the FDA to provide safety assurances. Our approach helps to design and clarify high level safety requirements that relate to the user interface of medical devices. It also supports the development of usage models for a range of interaction methods and their verification against safety requirements [15].

\subsection{Risk analysis}

The Royal Victoria Infirmary, a hospital in Newcastle, has developed a new system, called NIDUS, for treating babies with kidney problems for which — until now - there has been no suitable treatment. We have undertaken a risk analysis based on formal methods to show that this system is resilient to certain dangerous situations arising and our analysis is part of the process used to convince the regulator to allow the system to be used widely.

\subsection{Distributed Cognition for healthcare}

Distributed Cognition is a way of thinking about cognition as extending to the way artefacts are used in the world. It has been criticised for being difficult to understand and apply. We have made it more accessible by extending and refining the use of a tool, called DiCoT. We developed DiCoT as methodological support for applying Distributed Cognition to analyse healthcare contexts and medical device design and use. DiCoT has been applied in a variety of healthcare contexts, from hospital wards [3] to patient homes [14], and for a variety of different devices including infusion pumps, blood glucose meters and haemodialysis machines.

\section{UNDERSTANDING INCIDENTS}

If individuals fear the personal consequences of reporting incidents they are involved in, then they are less likely to be open about mistakes. Opportunities for learning from those incidents are lost, nothing is improved and similar incidents happen in the future. On the other hand if people feel that they will not be held responsible and only good will come from reporting incidents, then they will be much more likely to do so openly and honestly. Future harm is then avoided, preventing the future suffering of patients, their families and often the professionals involved who become secondary victims. Not only incidents but also 'near misses', where bad things almost happen, are more likely to be reported, allowing problems to be fixed before anyone is harmed at all. Furthermore, less time is wasted dealing with the consequences allowing nurses and doctors to focus on patient care. There is also a massive economic case: the UK National Health Service currently has set aside $£ 28$ billion for medical negligence liability costs alone. The benefits of a learning culture are enormous, but it needs to pervade all levels of the healthcare system for it to happen. A key issue facing healthcare is therefore how to embed a learning culture. CHI+MED has looked at how incidents are reported and investigated, developed new models to understand accidents, and looked at how media report incidents.

\subsection{The "Hot Cheese" model}

Poor design of medical devices has caused many incidents where patients have been harmed. However, design as such is not prominent in existing models of accident causation, particularly Reason's widely-used Swiss Cheese Model. The new "hot cheese model" highlights the impact of bad design on incidents in a simple, flexible and memorable way. The model sheds light on this hidden issue, and supports risk analysis and risk management in safety critical fields, including aviation, engineering, and healthcare. If it were adopted widely it could lead to further incidents being prevented, saving both lives and money for the healthcare system [7].

\subsection{Incident reporting and Investigation}

We identified serious flaws in the forms that UK healthcare professionals fill out after an incident that could affect the 
safety of a patient. They do not support easy and quick reporting at the point of care nor learning from incidents. We have developed a new form that encourages those filling it out to be more open and honest about mistakes. We have also developed techniques to support investigators and help understand the link between the theory and practical application of investigation methods.

\subsection{Responsible media reporting}

We have studied media reporting of incidents based on cases where babies died. The way the media report an incident can affect whether the underlying problem is corrected. Balanced reporting is key before investigations, such as coroner's reports or criminal investigations, are closed. The analysis suggests hospitals need to be open and honest rather than suggesting there is not a problem or that it is restricted to a single "bad seed", if they are to encourage reasoned reporting. They should make clear recommendations of how the hospital (or system) as a whole can change to prevent similar incidents occurring in the future [10].

\section{CONCLUSIONS}

Mobile health may be driven by attractive innovations in technology - wifi, tablets, cloud, internet of things, to name a few - but the CHI+MED project's broad experience of healthcare technologies (summarized in this paper) shows that innovation and safety are not so simply related. Healthcare is complex, and more complex in either mobile or home contexts, and especially when users are not trained clinicians - sometimes the patient's illness may even compromise safety using critical technologies to manage their health. New technologies can certainly enable new solutions, but they need implementing in thoughtful and informed ways if they are to be successful. The CHI+MED web site www.chimed.ac.uk provides numerous links to evidence and resources.

\section{ACKNOWLEDGEMENTS}

This work was funded by EPSRC (EP/G059063/1). It was conducted by a team of over 30 people (see www.chi-med.ac.uk/). Stakeholders (industry, healthcare) across many domains have also given their time.

\section{REFERENCES}

[1] D. P. Brumby, A. L. Cox, J. Back, and S. J. J. Gould. Recovering from an interruption: Investigating speed-accuracy tradeoffs in task resumption strategy. Journal of Experimental Psychology: Applied, 19(2):95-107, June 2013.

[2] J. Day, D. Furniss, and G. Buchanan. Meals and ingredients: Coping with compound resilience strategies. In Proceedings of the 6th Symposium of the Resilience Engineering Association, June 2015.

[3] D. Furniss, P. Masci, P. Curzon, A. Mayer, and A. Blandford. 7 themes for guiding situated ergonomic assessments of medical devices: A case study of an inpatient glucometer. Applied Ergonomics, 45:1668-1677, 2014.

[4] D. Furniss, A. A. O'Kane, R. Randall, S. Tavena, H. Mentis, and A. Blandford, editors. Fieldwork for Healthcare: Case Studies investigating Human Factors in Computing Systems. Synthesis lectures on assistive, rehabilitative and health-preserving technologies 5 . Morgan and Claypool, 2014.

[5] D. Furniss, A. A. O'Kane, R. Randall, S. Tavena, H. Mentis, and A. Blandford, editors. Fieldwork for Healthcare: Guidance for investigating Human Factors in Computing Systems. Synthesis lectures on assistive, rehabilitative and health-preserving technologies. Morgan and Claypool, 2015.

[6] Y. Li, P. Oladimeji, and H. Thimbleby. Exploring the effect of pre-operational priming intervention on number entry errors. In Proceedings of the 33rd Annual ACM Conference on Human Factors in Computing Systems (ACM SIG CHI 2015), pages 1335-1344. ACM, 2015.

[7] Y. Li and H. Thimbleby. Hot cheese: A processed swiss cheese model. The Journal of the Royal College of Physicians of Edinburgh, 44(2):116-121, June 2014.

[8] P. Masci, P. Curzon, and H. Thimbleby. Early identification of software causes of use-related hazards in medical devices. In Proceedings of the 5th EAI International Conference on Wireless Mobile Communication and Healthcare, October 2015.

[9] P. Masci, P. Oladimeji, P. Mallozzi, P. Curzon, and H. Thimbleby. PVSio-web: mathematically based tool support for the design of interactive and interoperable medical systems. In Proceedings of the 5th EAI International Conference on Wireless Mobile Communication and Healthcare, October 2015.

[10] C. Myketiak, S. Concannon, and P. Curzon. New/s design: Informing future design processes by understanding media reporting of medical errors with medical devices. In Proceedings of the 5th EAI International Conference on Wireless Mobile Communication and Healthcare, October 2015.

[11] G. Niezen. A continuous interaction approach to interactive medical device design. In MediCHI Workshop on Safer Interaction in Medical Devices at CHI 2013, April 2013.

[12] A. A. O'Kane. Using a third-wave HCI approach for researching mobile medical devices. Presented at the Workshop on HCI Research in Healthcare: Using Theory from Evidence to Practice at CHI 2014, 2014.

[13] P. Oladimeji, H. Thimbleby, P. Masci, and P. Curzon. Issues in number entry user interface styles: Recommendations for mitigation. In Proceedings of the 5th EAI International Conference on Wireless Mobile Communication and Healthcare, October 2015.

[14] A. Rajkomar, K. Farrington, A. Mayer, D. Walker, and A. Blandford. Patients' and carers' experiences of interacting with home haemodialysis technology: Implications for quality and safety. BMC Nephrology, 15(1):195, 2014.

[15] R. Rukšènas, P. Masci, and P. Curzon. Developing and verifying user interface requirements for infusion pumps: A refinement approach. In L. Petre and E. Sekerinski, editors, From Action Systems to Distributed Systems: The Refinement Approach. Chapman and Hall/CRC, In press.

[16] H. Thimbleby and A. Gimblett. Dependable keyed data entry for interactive systems. Electronic Communications of the EASST, 45: Formal Methods for Interactive Systems (FMIS 2011), June 2011. 